\title{
Laparoscopic Tool Tracking Method for Augmented Reality Surgical Applications
}

\author{
Alicia M Cano, Francisco Gayá, Pablo Lamata, Patricia Sánchez-González, \\ and Enrique J. Gómez \\ Grupo de Bioingeniería y Telemedicina (GBT), \\ ETSIT, Universidad Politécnica de Madrid \\ C/ Ciudad Universitaria s/n, 28040 Madrid, Spain
}

\begin{abstract}
Vision-based tracking of laparoscopic tools offers new possibilities for improving surgical training and for developing new augmented reality surgical applications. We present an original method to determine not only the tip position, but also the orientation of a laparoscopic tool respect to the camera coordinate frame. A simple mathematical formulation shows how segmented tool edges and camera field of view define the tool 3D orientation. Then, 3D position of the tool tip is determined by image 2D coordinates of any known point of the tool and by tool's diameter. Accuracy is evaluated in real image sequences with known ground truth. Results show a positioning error of 9,28 mmRMS, what is explained by inaccuracies in the estimation of tool edges. The main advantage of proposed method is its robustness to occlusions of the tool tip.
\end{abstract}

Keywords: laparoscopic tool tracking, video-endoscopic analysis, augmented reality tools, surgical training programs.

\section{Introduction}

Laparoscopic surgery involves an important challenge for surgeons in getting used to the reduced workspace and the limited sensory interaction This technique offers a different interaction paradigm to the traditional open approach, based on the distance manipulation of specialized surgical instruments and an indirect visualization of the surgical scene captured by an endoscope. This makes the learning and developing of the technique a difficult task.

3D localization of surgical instruments opens the possibility of multiple applications for overcoming some of the current limitations of laparoscopy. Surgical training programs lack of standardized and assessed methods with objective metrics to guarantee the complete instruction of surgeons The analysis of trajectory and movements of tools offers objectives parameters, suitable for defining objective metrics

In particular, new augmented reality devices for training can exploit the benefits of this tracking to provide trainees with constructive and effective feedback about their performance 
On the other hand, the last advances on augmented reality applied in surgery provide useful information for image-guidance for intra-operative procedures in minimally invasive surgery. In these techniques, the knowledge of the tools 3D position constitutes an important step to guide and advice surgeon about the proximity of delicate areas

In this work we address the analysis of laparoscopic video sequences as the main source of information to get 3D information of the surgical scene. We aim to achieve $3 \mathrm{D}$ tracking of laparoscopic tools using the $2 \mathrm{D}$ information of the image. This is an interesting alternative to other means to localize instruments based on the use of position sensor on the tools (optical, electromagnetic or mechanical), which can be very bulky

In the scientific literature, a previous approach uses a LED placed in the tool tip that projects laser dots onto the surface of the organs. The optical markers are then detected in the endoscope image on the surface to allow localizing the instrument with respect to the scene. Other more recent works have tackled the problem without extra markers. Tonet proposed a heuristic approach to estimate the depth of the instrument by means of the knowledge of the tool width and the orientation of its edges. Authors have previously formalised two methods for assessing the 3D position of the tools' tips The first uses the vanishing point of the tool in the image, and the second one the tool width at the tip in the image.

Video-based localization of laparoscopic tools requires two main steps: extraction of relevant $2 \mathrm{D}$ information from the image, and calculation of the $3 \mathrm{D}$ coordinates of the tool's tip. This paper focuses on the second, and contributes with a new method that calculates not only tool's position, but also orientation. The method is also robust to the occlusion of the tool tip.

\section{Materials and Methods}

The $2 \mathrm{D}$ relevant information is composed by the tool edges and tip, which are segmented from laparoscopic images. Tool edges detection strategy is based on the temporal continuity between consecutives frames and edge detector operator (Sobel). Colour analysis of the laparoscopic tool allows us to identify a point of the tool tip.

The second stage of the proposed method is explained in detail in the next section.

\subsection{D Localization Method for Laparoscopic Tool}

Segmented tool edges and camera field of view (FoV) define the tool 3D orientation. Then, 3D position of the tool tip is determined by image 2D coordinates of the tool tip (or any characteristic point of the tool as we explain later) and by tool's physical diameter ( 5 or $10 \mathrm{~mm}$ usually in laparoscopic tools).

Let $\mathrm{C}$ be the optical camera centre, $\mathrm{P}$ a point of the laparoscopic tool axis, and $\mathrm{u}_{\mathrm{CP}}$ unitary vector from $C$ to $P$. Let $\Pi$ be the image plane, and $\left(\Omega_{1}, \Omega_{2}\right)$ the tangential planes to the tool containing $C$. The intersection of both $\left(\Omega_{1}, \Omega_{2}\right)$ planes with $\Pi$ plane results in $E_{1}$ and $E_{2}$, the two projective edges of the tool (see fig 1.a), being $\left(\vec{u}_{E 1}, \vec{u}_{E 2}\right)$ their unitary vectors.

Let $\left(\vec{u}_{C E 1}, \vec{u}_{C E 2}\right.$ ) be unitary vectors from $\mathrm{C}$ to any point of $\mathrm{E}_{1}$ or $\mathrm{E}_{2}$ respectively. Each $\Omega_{\mathrm{i}}$ plane normal vector, $\left(\vec{u}_{\Omega 1}, \vec{u}_{\Omega 2}\right)$, is obtained from: 


$$
\vec{u}_{\Omega i}=\vec{u}_{E i} \times \vec{u}_{C E i}, i=1,2
$$

because both vectors are included in $\Omega_{\mathrm{i}}$ plane.

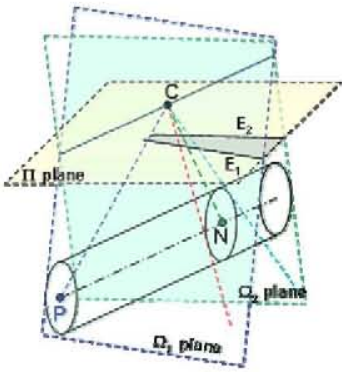

(a)

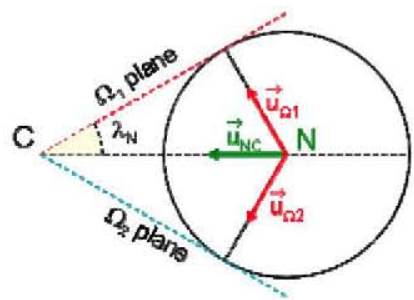

(b)

Fig. 1. (a) Projective model of the laparoscopic tool; C: optical center, P: tip of the laparoscopic tool; $\mathrm{N}$ : point of the cylinder axis which projective line is perpendicular to this axis; $\Omega_{1}, \Omega_{2}$ : planes of sight of the tool edges; $\Pi$ plane: image plane, E1, E2: projective tool edges, (b) Transversal section of the laparoscopic tool at $\mathrm{N}$ point

The perpendicular plane to the cylinder's axis that contains $\mathrm{C}$ defines a circle section of the cylinder as shown in fig. 1.b. Let $\mathrm{N}$ be the centre of this circle, and $2 \lambda_{\mathrm{N}}$ the angle between planes $\Omega_{1}$ and $\Omega_{2}$.

As shown in fig. 1.b the addition $\vec{u}_{\Omega 1}$ and $\vec{u}_{\Omega 2}$, results in the direction of $\overline{C N}$, and its cross product gives the tools' direction $\vec{u}_{N P}$. Therefore angle $\lambda_{\mathrm{N}}$ is expressed like:

$$
\tan \lambda_{N}=\frac{\left|\vec{u}_{\Omega 1}+\vec{u}_{\Omega 2}\right|}{\left|\vec{u}_{\Omega 1}-\vec{u}_{\Omega 2}\right|}
$$

Equally, it is possible to determine $\mathrm{ICNI}$ from the tool radius $\left(\mathrm{r}_{\mathrm{CYL}}\right)$ :

$$
|C N|=r_{C Y L} / \sin \lambda_{N}
$$

Finally, CP is found with:

$$
\overline{C P}=\frac{|C N| \cdot \vec{u}_{C P}}{\vec{u}_{C P} \cdot \vec{u}_{C N}}
$$

Note that $\mathrm{P}$ can be any point of the tool, it only requires to be identified in the image. And as $\vec{u}_{N P}$ is known, any other point of the tool P' (the tip) can be obtained by knowing the physical distance between $\mathrm{P}$ and P'.

\subsection{Validation Workbench}

Laboratory laparoscopic setting has been built to simulate the real surgical geometry and the range of movements of the tool. This scenery allows us to determine the ground truth of the tool positions thanks to the introduction of a background plane (a grid of points) and an inclined board $\left(30^{\circ}\right)$. This board is placed at a known distance 
from the optical centre point, knowing the position of any point of the plane respect to the camera. This way the 3D trajectory of the tool keeping the tip alongside this board is defined with only $2 \mathrm{D}$ information gathered from the segmentation stage.

Two validation sequences are acquired, performing two different movements: one with a constant depth ("Constant depth" sequence) and the second one with a variable depth ("Variable depth" sequence). Each sequence has about 275 frames.

\section{Results}

The two lab sequences have been analysed with proposed method (we call it Transversal Section based method) and with two previous ones based on the Vanishing Point and on the Apparent Diameter. Accuracy of assessed 3D coordinates is given by comparing them to the Ground Truth known by the $2 \mathrm{D}$ position of the tool in the image. Results are reported in table 1 and fig 2.

Table 1. Error characterization of methods for validation sequences. Mean error and standard deviation (SD), in $\mathrm{mm}$.

\begin{tabular}{cccccc}
\hline & \multicolumn{2}{c}{ Constant depth } & \multicolumn{2}{c}{ Variable depth } \\
\hline & Axis & Mean & SD & Mean & SD \\
\hline \multirow{2}{*}{ Vanishing } & $\mathrm{X}$ & 2.29 & 1.81 & 0.98 & 7.2 \\
Point & $\mathrm{Y}$ & 3.43 & 11.09 & 1.39 & 3.42 \\
& $\mathrm{Z}$ & 42.16 & 22.50 & 33.22 & 9.97 \\
\hline \multirow{2}{*}{ Apparent } & $\mathrm{X}$ & 0.71 & 0.15 & 0.60 & 0.76 \\
Diameter & $\mathrm{Y}$ & 1.93 & 0.86 & 0.24 & 3.55 \\
& $\mathrm{Z}$ & 2.39 & 2.61 & 6.32 & 4.36 \\
\hline \multirow{2}{*}{ Transversal } & $\mathrm{X}$ & 0.08 & 0.08 & 0.03 & 1.4 \\
Section & $\mathrm{Y}$ & 0.35 & 1.25 & 1.54 & 3.66 \\
& $\mathrm{Z}$ & 2.03 & 2.09 & 8.65 & 3.55 \\
\hline
\end{tabular}

Root Mean Square Error

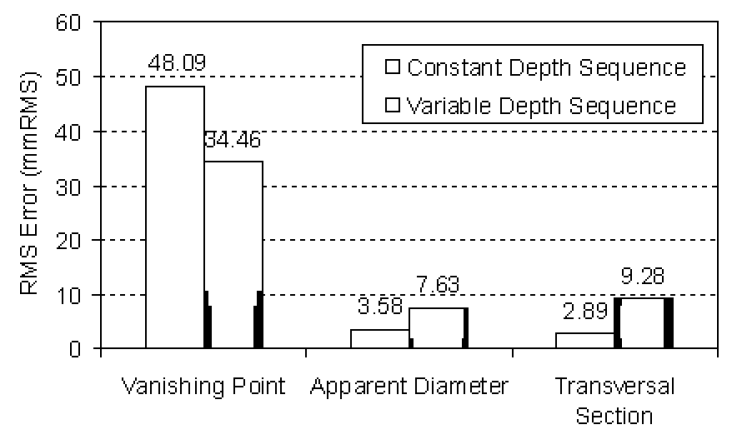

Fig. 2. Root Mean Square Error (mmRMS) in Z coordinates for three methods 


\section{Discussion}

A new method for video-based tracking of laparoscopic tool has been proposed. It is robust to tip occlusions providing that a visible characteristic point of the tool is visible in the image. It determines not only the tip position, but also the orientation of a laparoscopic tool respect to the camera coordinate frame.

Validation sequences in a controlled laboratory setting were taken to enable the estimation of the ground truth of tool's positions. This process might be subjected to biases due to errors in calibration or in the image segmentation of the tool. Nevertheless, we think this practical solution is valid to present the relative performance of the three methods and a first approximation of its tracking accuracy. Our future work will consider the acquisition of a reliable ground truth and the use of barrel distortion correction in laparoscopic sequences to get an accurate estimation of the performance of these video-based tracking methods.

Results have verified the potential of video-based tracking methods, as already concluded in previous works Proposed method offers a good accuracy (error of 2,89 mmRMS and 9,28 mmRMS for the each of the two sequences, see fig.2). The origin of this error is explained with possible calibration issues.

Measured accuracy of proposed Transversal Section method does not improve what the Apparent diameter method achieves (see fig.2). Nevertheless, the latest requires a visualization without obstacles of the tool tip, limiting its potential use respect to proposed method. Moreover, a combination of both methods could improve the behaviour of each other, since the $3 \mathrm{D}$ localization is determined through different features of the tool.

A limiting factor in image-based tracking of tools is the presence of blurred tool's edges caused by its movement. This causes an inaccurate edge detection, and an error in a later 3D pose estimation. A robust edge detection can include temporal constrains, an also geometrical constrains based on the position and projection of the insertion point (trocar point) what is easily found with the 3D orientation information extracted from proposed method.

Current tracking performance is good enough for gesture analysis and an objective evaluation of surgical manoeuvres, where non real time is needed and accuracy around $3 \mathrm{~mm}$. is enough to determine objective metrics. An improvement of the method addressing the points commented before will increase its potential applications in the clinical routine. In particular, this tools tracking appears as one of the keys to development of new augmented reality tools for laparoscopic surgery.

\section{Conclusion}

This work proposes a new method for video-based tracking of laparoscopic tools that obtains both their position and orientation. It is also robust to occlusions of the tip equipping the tool with an additional landmark.

Results in laboratory environment have validated the proposed method as an alternative to traditional tracking, with important applications such as an objective evaluation of motor skills and the development of augmented reality environments. 


\section{References}

Fuchs, K.H.: Minimally Invasive Surgery. Endoscopy 34, 154-159 (2002)

Darzi, A., Smith, S., Taffinder, N.: Assessing operative skill: needs to become more objective. BMJ 316, 1917-1918 (1998)

Rosen, J., Brown, J.D., Chang, L., Sinanan, M.N., Hannaford, B.: Generalized Approach for Modeling Minimally Invasive Surgery as a Stochastic Process Using a Discrete Markov Model. IEEE Transactions on Biomedical Engineering 53(3), 399-413 (2006)

Aggarwal, R., Grantcharov, T., Moorthy, K., Milland, T., Papasavas, P., Dosis, A., Bello, F., Darzi, A.: An evaluation of the feasibility, validity and reliability of laparoscopic skills assessment in the operating room. Ann. Surg. 245(6), 992-999 (2007)

Blum, T., Sielhorst, T., Navab, N.: Advanced augmented reality feedback for teaching 3D tool manipulation. New Tech. frontiers in minimally invasive therapies, pp. 223-236 (2007)

Fuchs, K.H., Livingston, M.A., Raskar, R., D'nardo, C., Keller, K., State, A., Crawford, J.R., Rademacher, P., Drake, S.H., Meyer, A.A.: Augmented Reality Visualization for laparoscopic surgery. In: Wells, W.M., Colchester, A.C.F., Delp, S.L. (eds.) MICCAI 1998. LNCS, vol. 1496, pp. 934-943. Springer, Heidelberg (1998)

Immersion: Digiziting, http://www.immersion.com/digitizer/

Krupa, A., Gangloff, J., Doignon, C., de Mathelin, M., Morel, G., Leroy, J., Soler, L., Marescaux, J.: Autonomous 3-D positioning of surgical instruments in robotized laparoscopic surgery using visual servoing. IEEE Trans. on Robo. and Auto. 19(5), 842-853 (2003)

Tonet, O., Armes, T.U., Magali, G., Dario, P.: Tracking Endoscopic Instruments Without Localizer: Image Analysis-Based Approach Stud. Health Tech. Inf. 119, 544-549 (2005) Cano, A.M., Lamata, P., Gayá, F., del Pozo, F., Gómez, E.J.: New Methods for VideoBased Tracking of Laparoscopic Tools. In: Harders, M., Székely, G. (eds.) ISBMS 2006. LNCS, vol. 4072, pp. 142-149. Springer, Heidelberg (2006)

Voros, S., Orvain, E., Cinquin, P.: Automatic detection of instruments in laparoscopic images: a first step towards high level command of robotized endoscopic holders. In: BIOROB 2006 1st IEEE/RAS-EMBS Int. Conf. On Biomed. Rob. and Biomecha., Pisa (2006) 